\title{
CIRCULACIÓN DE NUTRIENTES EN ALGUNOS ECOSISTEMAS FORESTALES DEL MONTSENY (BARCELONA) ${ }^{1}$
}

\author{
por \\ Ll. Ferrés², F. Rodà2, A.M.C. Verdú ${ }^{3} \&$ J. Terradas ${ }^{2}$
}

\section{SUMMARY}

Individual stands of evergreen-oak (Quercus ilex), beech (Fagus sylvatica), and fir (Abies alba) in the Montseny mountains (NE Spain) are compared in terms of nutrient fluxes in bulk precipitation, throughfall, and litterfall. Precipitation washes out a greater amount of nutrients from the fir canopy than from either the evergreen-oak or beech canopies. More $\mathrm{K}^{+}$than $\mathrm{Ca}^{2+}$ is washed out from the evergreen-oak canopy, while the reverse is true for beech and fir. Dry weight of litterfall and nutrient return in litterfall were: evergreen-oak $>$ beech $>$ fir. Our beech stand markedly differs from the other European beech forests because of its much lower $\mathrm{N}$ flux in litterfall, probably indicating a lower availability of $\mathrm{N}$ in this southern mountain.

Above-ground plant biomass, production, and nutrient contents were measured in the evergreen-oak stand. Tree leaf biomass is $6.1 \mathrm{t} \mathrm{ha}^{-1}$, a higher figure than those commonly found in temperate deciduous forests. Nutrient concentrations in evergreen-oak wood are relatively high. Therefore, plant nutrient contents $\left(\mathrm{Kg} \mathrm{ha}^{-1}\right)$ are also relatively high, in spite of moderate biomass. Large amounts of $\mathrm{Ca}$ accumulate in the bark of this stand, developed on silicate bed-rock. Living twigs and branches of evergreen-oak could act as a store of nutrients, counteracting the fluctuations in soil nutrient availability brought about by erratic rainfall patterns. This trait should be adaptive under Mediterranean climates.

\section{RESUMEN}

Se comparan las pautas de circulación de nutrientes en la deposición global, la trascolación, y el desfronde de un encinar montano, un hayendo, y un abetal del Montseny. Las precipitaciones lavan mucho más nutrientes de las copas del abetal que de las del encinar y el hayedo. En el encinar se lava mucho más $\mathrm{K}^{+}$que $\mathrm{Ca}^{2+}$, mientras que en los otros dos bosques sucede al contrario. Las cantidades de desfronde (peso seco) y en los flujos de nutrientes en el desfronde resultaron ser: encinar $>$ hayedo $>$ abetal. El hayedo del Montseny se diferencia de los demás hayendos europeos por sus reducidos aportes de $\mathrm{N}$ en el desfronde, indicando quizá una menor disponibilidad de $\mathrm{N}$ en este macizo meridional.

En el encinar montano se midieron además la biomasa, producción, y mineralomasa aéreas. La biomasa arbórea de hojas es de $6.1 \mathrm{tha}^{-1}$, cifra superior a la encontrada normalmente en bosques caducifolios templados. Las concentraciones de nutrientes en la madera de encina son relativamente altas. Por este motivo, las mineralomasas del rodal son también elevadas, a pesar de que la biomasa es sólo moderada. Destaca la importancia de la corteza como acumuladora de $\mathrm{Ca}$. Las ramas y ramitas vivas de encina podrían actuar como reserva de nutrientes que amortiguaría las fluctuaciones en la disponibilidad de elementos esenciales. Tal capacidad resultaría adaptativa ante el régimen irregular de pluviosidad de los climas mediterráneos.

(1) El presente trabajo compara algunos resultados de diversos proyectos de investigación que han recibido financiaciación de distintas fuentes. Debemos expresar nuestro agradecimiento al Comité Conjunto Hispano-Norteamericano de Cooperación Científica y Tecnológica (COOP-370 sobre el encinar), «Servei de Parcs Naturals de la Diputació de Barcelona» (hayedo y abetal) y «Serveis de Protecció de la Natura de la Generalitat de Catalunya» (infraestructura de la zona experimental de la Castanya).

(2) Departament d'Ecologia. Facultat de Ciències. Universitat Autònoma de Barcelona. Bellaterra (Barcelona).

(3) Departament de Botànica. Facultat de Ciències. Universitat Autònoma de Barcelona.

Dirección actual: Departament de Biologia. Escola d'Enginyeria Tècnica Agricola i d'Especialitats agropecuàries de Barcelona. Urgell, 187. Barcelona-11. 


\section{INTRODUCCION}

La circulación de nutrientes es uno de los aspectos clave de la dinámica de los ecosistemas terrestres, y recibe atención creciente como manifestación integradora de la conducta de los sistemas ecológicos, ya sea en condiciones naturales o bien sometidos a perturbaciones experimentales (Harwell et al. 1977, Likens et al. 1970, O’Neill et al. 1977, Van Voris et al. 1980, Webster et al. 1975).

Las pautas de circulación de los elementos nutritivos se conocen con cierto detalle en los ecosistemas forestales, en especial en las regiones de clima templado. Cole \& Rapp (1981) han examinado las regularidades que se observan en dichas pautas, así como las similitudes y diferencias entre biomas, utilizando para ello los datos de 32 rodales del hemisferio norte. Sólo uno de dichos rodales corresponde a un bosque mediterráneo (el encinar de Rouquet (Montpellier), Lossaint \& Rapp 1978), situación que contrasta con la cantidad de información disponible sobre los bosques caducifolios y de coníferas extramediterráneos.

El objetivo de este artículo es presentar los resultados obtenidos durante el estudio de la circulación de nutrientes en 3 bosques del parque natural y reserva de la biosfera del Montseny (Barcelona-Girona): un encinar montano, un hayedo, y un abetal. El gradiente altitudinal del Montseny ofrece la posibilidad de comparar estos tres tipos de bosque tan contrastados, que se encuentran aquí a poca distancia unos de otros. El presente estudio contribuye a paliar la escasez de datos biogeoquímicos referentes a Quercus ilex y a Abies alba, y permite comparar la circulación de nutrientes en los hayedos del Montseny, cercanos a uno de sus límites meridionales de distribución, con la de los hayedos centroeuropeos estudiados por diversos autores.

\section{AREAS DE ESTUDIO}

El Montseny se encuentra a $40 \mathrm{~km}$. al NNE de Barcelona, y sus características generales se describen en la Memoria Informativa del Plan Especial del Parque Natural del Montseny (Anónimo 1976). Bolòs (1983) ha estudiado la vegetación del macizo.

Los trabajos del presente artículo se realizaron dentro de una parce- 
la experimental para cada uno de los 3 bosques estudiados. Los 3 rodales son bosques altos irregulares.

\section{Encinar montano}

La parcela de encinar $\left(41^{\circ} 46^{\prime} \mathrm{N}, 2^{\circ} 21^{\prime} \mathrm{E}\right)$ tiene 0.23 ha y se encuentra en el valle de La Castanya, a $665 \mathrm{~m}$ s.n.m. Está situada al pie de una vertiente abrupta $\left(30^{\circ}\right)$. La pendiente dentro de la parcela es mucho más suave, variando entre 7 y $23^{\circ}$. La orientación es W y NW. La roca madre es una pizarra (filita) metamórfica. El suelo, de carácter coluvial, es una tierra parda, muy pedregosa, de textura franca, con un $\mathrm{pH}$ en agua de 4.7-5.2, un contenido de materia orgánica del 3.4-6.5\%, una relación $\mathrm{C} / \mathrm{N}$ de 8.5-13.0, y una capacidad de intercambio catiónico de 0.19-0.26 eq $\mathrm{kg}^{-1}$. La precipitación media anual es de $900 \mathrm{~mm}$, y la temperatura media anual es de $9-10^{\circ} \mathrm{C}$. El estrato arbóreo es denso, y está formado exclusivamente por Quercus ilex $\mathrm{L}$. El área basal era de $26.6 \mathrm{~m}^{2} \mathrm{ha}^{-1}$ en 1979. Hay 2010 pies ha ${ }^{-1}$ de DN (diámetro normal) $>5 \mathrm{~cm}$, y 536 pies $\mathrm{ha}^{-1}$ de DN $>15 \mathrm{~cm}$. El diámetro medio de los pies de DN $>15 \mathrm{~cm}$. es de $18.6 \mathrm{~cm}$. Los árboles dominantes tienen una altura de $9-12 \mathrm{~m}$ y su edad se estima en 60-90 años. El estrato arbustivo es escaso, y está dominado por pies pequeños de encina, con algunos ejemplares aislados de Erica arborea, Crateagus monogyna, y Phyllirea media. El estrato herbáceo es también escaso, y está formado por Hedera helix, Rubus ulmifolius, Brachypodium sylvaticum, Melica uniflora, Pteridium aquilinum, Polystichum setiferum, Asplenium adiantum-nigrum, Teucrium scorodonia, Hepatica nobilis, Fragaria vesca, y otras especies. El sector de la parcela que tiene menor pendiente estuvo cultivado en el pasado, y el encinar que se desarrolló tras el abandono de los cultivos no ha sido talado nunca, como evidencia la ausencia casi total de encinas de rebrote. En el sector de mayor pendiente, que no fue cultivado, el último aclareo parece haber tenido lugar hace unos 45 años.

\section{Hayedo}

La parcela de hayedo $\left(41^{\circ} 46^{\prime} \mathrm{N}, 2^{\circ} 28^{\prime} \mathrm{E}\right)$ tiene 0.12 ha y se encuentra en el valle de Santa Fe, a 1.165 m s.n.m. Está situada en un interfluvio de escasa pendiente $\left(5^{\circ}\right)$. La roca madre es una granodiorita, profundamente alterada. El suelo es una tierra parda lixiviada, de textura arenosa, con un $\mathrm{pH}$ en agua de 4.5-5.0, un contenido de materia orgánica del 1.0-5.3\%, una relación $\mathrm{C} / \mathrm{N}$ de 6.1-11.3, y una capacidad de intercambio 
catiónico de 0.16-0.24 eq $\mathrm{kg}^{-1}$. La precipitación media anual es de unos $1.200 \mathrm{~mm}$ (Rodà 1983), y la temperatura media anual es de $8-9^{\circ} \mathrm{C}$. El estrato arbóreo es denso, y está formado por Fagus sylvatica L., con algún ejemplar aislado de Ilex aquifolium. El área basal era de $26.7 \mathrm{~m}^{2}$ $\mathrm{ha}^{-1}$ en 1980 . Hay 1.460 pies ha ${ }^{-1}$ de DN $>5 \mathrm{~cm}$, y 625 pies ha-1 de DN $>15 \mathrm{~cm}$. El diámetro medio de los pies de DN $>15 \mathrm{~cm}$ es de $20.7 \mathrm{~cm}$. Los árboles dominantes tienen una altura de $16-20 \mathrm{~m}$, y una edad de 5070 años. El último aclareo se realizó hace 15-30 años. El estrato arbustivo es muy escaso, y está formado principalmente por pies pequeños de haya. El estrato herbáceo es también pobre, con Festuca ovina, Pteridium aquilinum, y Anemone nemorosa como especies principales.

\section{Abetal}

La parcela de abetal $\left(41^{\circ} 47^{\prime} \mathrm{N}, 2^{\circ} 27^{\prime} \mathrm{E}\right)$ forma parte del abetal de Passavets, en la cabecera del valle de Santa Fe. Tiene 0.12 ha, y se encuentra en una pendiente de $29^{\circ}$ orientada al NNW, a una altitud de $1.355 \mathrm{~m}$. La roca madre es una corneana. El suelo, muy pedregoso, corresponde a un ránker. La precipitación anual media es cercana a los $1.200 \mathrm{~mm}$, y la temperatura media anual es de $7-8^{\circ} \mathrm{C}$. El estrato arbóreo es denso, y está formado exclusivamente por Abies alba Mill. El área basal era de $42.4 \mathrm{~m}^{2} \mathrm{ha}^{-1}$ en 1980 . Hay 567 pies ha ${ }^{-1}$ de DN $>5 \mathrm{~cm}$, y 467 pies ha ${ }^{-1}$ de $\mathrm{DN}>15 \mathrm{~cm}$. El diámetro medio de los árboles de $\mathrm{DN}>15$ $\mathrm{cm}$ es de $31.8 \mathrm{~cm}$. Los abetos dominantes tienen una altura de $21-25 \mathrm{~m}, \mathrm{y}$ una edad de 120-160 años. Los árboles son pues muchos mayores y más viejos en el abetal que en el encinar y el hayedo. Esto se debe en parte a que el último aclareo tuvo lugar en el abetal hace unos 70 años. El estrato arbustivo es prácticamente nulo; el herbáceo es muy pobre y contiene Festuca ovina, Deschampsia flexuosa, Hepatica nobilis, y Polypodium vulgare como especies principales. Hay un estrato muscinal discontinuo, dominado por Hylocomium splendens.

\section{METODOS}

La mayor parte de los métodos utilizados en el encinar se describen en Ferrés et al. (1980), Rodà et al. (1980), Verdú et al. (1980) y Terradas \& Escarré (1983). En el hayedo y el abetal se utilizaron los mismos métodos.

La caída de ramas gruesas (diámetro basal $>0.5 \mathrm{~cm}$ ) se estudió en 
cada parcela mediante 4 rectángulos permanentes de $10 \times 1 \mathrm{~m}$. La producción de hojas y frutos se consideró igual al desfronde de las fracciones respectivas. Las producciones de madera y de corteza se obtuvieron a partir de las regresiones de biomasa sobre $\mathrm{DN}$, y de las tasas anuales de incremento radial obtenida midiendo los anillos de crecimiento en cortes de madera al microtomo teñidos de picrofucsina, según su adaptación del método descriptivo por Susmel et al. (1976).

En las muestras de aguas, $\mathrm{NO}_{3}^{-}$y $\mathrm{PO}_{4}^{3-}$ se analizaron con un autoanalizador Technicon siguiendo métodos estándard. $\mathrm{El} \mathrm{Cl}^{-}$se analizó por colorimetría con el método del tiocianato mercúrico. El pH se determinó electrométricamente. Los $\mathrm{pH}$ se promediaron transformándolos a concentraciones de hidrogeniones, ponderando éstas por volumen, y reconvirtiendo el resultado a $\mathrm{pH}$. En las muestras vegetales, el $\mathrm{N}$ se analizó por el método Kjeldahl, y el $\mathrm{P}$ mediante digestión ácida seguida de colorimetría por el método del vanado-molibdofosfórico.

Algunos de los resultados presentados en este artículo para el encinar montano difieren ligeramente de los publicados con anterioridad (Ferrés et al. 1982, Rodà et al. 1980, Verdú et al. 1980), debido a que los períodos de muestreo considerados son distintos, y a que se dispone de resultados de nuevos análisis.

\section{RESULTADOS Y DISCUSION}

\section{Disposición global}

La disposición global (bulk precipitation (Whitehead \& Feth 1964), bulk deposition) es la mezcla de deposición húmeda (precipitation) y de sedimentación gravitatoria de partículas que se recoge en los colectores permanentemente abiertos a la atmósfera, como los utilizados en el presente estudio.

Los aportes anuales de nutrientes en la deposición global son bastante parecidos en los 3 bosques estudiados (tabla 1). Las principales diferencias estriban en que los aportes de $\mathrm{Na}^{+}$y de $\mathrm{Cl}^{-}$son más elevados en el hayedo que en los otros dos bosques, y en que los aportes de ácido $\left(\mathrm{H}^{+}\right)$son mayores en el Valle de Santa Fe (hayedo y abetal) que en La Castanya (encinar). Como se indica en las notas, los aportes de la tabla 1 


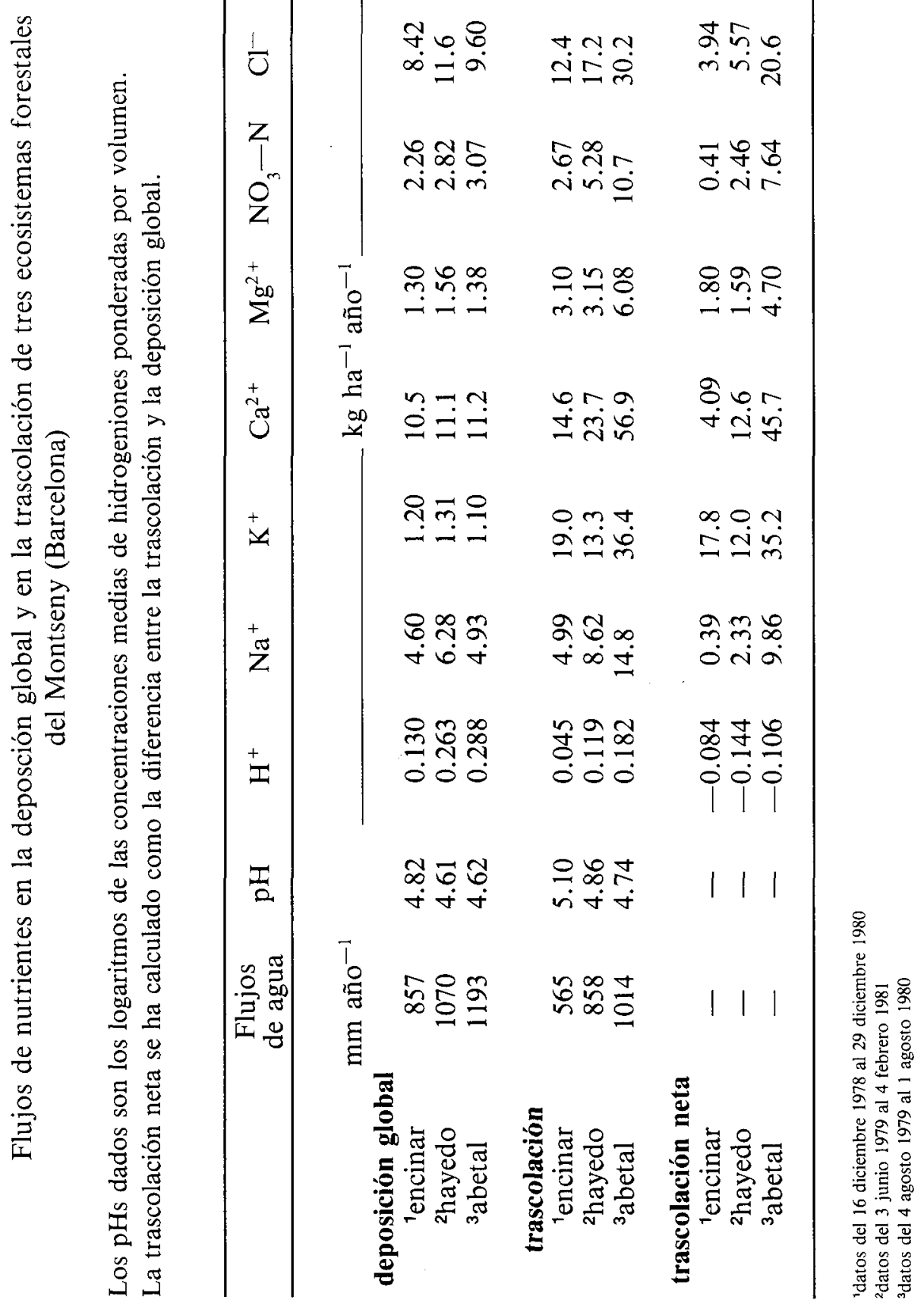


se refieren a distintos períodos de muestreo para cada bosque. Cuando se calculan los resultados correspondientes a períodos comunes, los aportes de todos los iones analizados son bastantes mayores en el hayedo que en

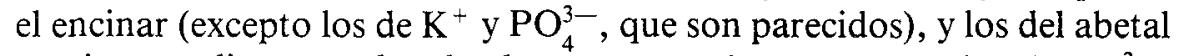
son intermedios entre los dos bosques anteriores (excepto los de $\mathrm{PO}_{4}^{3-}$, que son menores). El hecho de que los aportes sean mayores en el hayedo que en el encinar se debe a que la pluviosidad es más elevada en el hayedo (1.070 v. $724 \mathrm{~mm}$ año ${ }^{-1}$, durante el período común), y no a que existan diferencias importantes en las concentraciones iónicas en la deposición global de ambos lugares (excepto para $\mathrm{H}^{+}$, cuya concentración durante el período común es un $33 \%$ más alta en el hayedo).

En conjunto, la deposición global del Montseny no destaca especialmente por los aportes de los iones analizados respecto a muchas otras localidades de Europa y Norteamérica (cf. Steinhardt 1973). La deposición global del Montseny es rica en $\mathrm{Ca}^{2+}\left(11 \mathrm{~kg} \mathrm{ha}^{-1}\right.$ año-1), pero ésta es una situación generalizada en Europa occidental (Allen et al. 1968, Granat 1972, Steinhardt 1973). Los aportes $\mathrm{PO}_{4}-\mathrm{P}$ en la deposición global del Montseny (tabla 1) son bajos. Los ecosistemas forestales del Montseny se benefician anualmente de $2-3 \mathrm{~kg} \mathrm{ha}^{-1}$ de $\mathrm{NO}_{3}-\mathrm{N}$ (tabla 1). El $\mathrm{NO}_{3}-\mathrm{N}$ representa a menudo sólo un $25-30 \%$ del $\mathrm{N}$ total de la deposición global (Matzner et al. 1982, Verry \& Timmons 1977), correspondiendo el resto a $\mathrm{NH}_{4}-\mathrm{N}$ y a $\mathrm{N}$ orgánico. Esto indicaría que los aportes de $\mathrm{N}$ total en la deposición global del Montseny pueden ser de 8-12 kg $\mathrm{ha}^{-1}$ año ${ }^{-1}$. Este flujo pasivo de entrada de $\mathrm{N}$ constituye un subsidio para el ecosistema, en forma de fertilización crónica de bajo nivel. Abundan en la bibliografía los ejemplos de aportes de $\mathrm{N}$ atmosférico parecidos o mayores que los estimados para el Montseny. Así, Rapp (1969) encontró una media de $14.0 \mathrm{~kg} \mathrm{ha}^{-1}$ año ${ }^{-1}$ de $\mathrm{N}$ Kjeldahl disuelto $\left(\mathrm{NH}_{4}\right.$ más $\mathrm{N}$ orgánico disuelto) en el sur de Francia. Matzner et al. (1982) obtuvieron una media de $24.7 \mathrm{~kg} \mathrm{ha}^{-1}$ año ${ }^{-1}$ de $\mathrm{N}$ total en la deposición global de los bosques de Solling (Alemania).

En la deposición global del Montseny, la concentración de $\mathrm{Na}^{+}$está altamente correlacionada con la de $\mathrm{Cl}^{-}$, y ambos iones se encuentran en proporciones muy parecidas a la que existe en el agua de mar. Esto indica el origen predominantemente marino del $\mathrm{Na}^{+}$y del $\mathrm{Cl}^{-}$en las localidades estudiadas. Suponiendo que todo en $\mathrm{Na}^{+}$de la deposición global del Montseny fuese de origen marino y que no hubiese fraccionamiento entre los distintos iones, y utilizando los cocientes iónicos en las medias 
ponderadas de la deposición global y en el agua de mar, puede calcularse que el $90-95 \%$ del $\mathrm{Cl}^{-}$, el $43-48 \%$ del $\mathrm{Mg}^{2+}$, el $14-27 \%$ del $\mathrm{K}^{+}$, y el 1.7-2.2\% del $\mathrm{Ca}^{2+}$ aportados por la deposición global son de origen marino. Es decir, $\mathrm{K}^{+}$y $\mathrm{Ca}^{2+}$ son fundamentalmente continentales, mientras que el $\mathrm{Mg}^{2+}$ es de origen mixto, continental y marino. Los mayores aportes de $\mathrm{Na}^{+}$y Cl$^{-}$en el hayedo (tabla 1) reflejan pues una mayor influencia marítima en este rodal, debida seguramente a la falta de barreras topográficas importantes entre la parcela del hayedo y el litoral mediterráneo, que se encuentra a sólo $21 \mathrm{~km}$ en línea recta. Las parcelas del abetal y del encinar están orientadas de espaldas al mar, lo cual reduciría la circulación del aire marítimo en su vertical, disminuyendo por consiguiente los aportes de $\mathrm{Na}^{+}$y $\mathrm{Cl}^{-}$.

Como se observa en la tabla 1, la deposición global del Montseny es actualmente ácida. Debido a la falta de datos, se desconocía hasta ahora la existencia de lluvias ácidas en la región mediterránea, creyéndose que este problema ambiental se limitaba en Europa a los paises del centro y del norte del continente y a las islas británicas (Likens et al. 1979). La causa principal de la acidez de las precipitaciones es la oxidación de los óxidos de azufre y de nitrógeno emitidos a la atmósfera como contaminantes (Likens \& Bormann 1974, Overrein et al. 1981). Existe una abundante bibliografía sobre los efectos de las precipitaciones ácidas en los ecosistemas terrestres y acuáticos (Dochinger \& Seliga 1976, Drabløs \& Tollan 1980, Hutchinson \& Havas 1980, Ulrich \& Pankrath 1983), y es sabido que la sensibilidad de los suelos y de las aguas de escorrentía a la acidificación varía mucho de un lugar a otro. Los ecosistemas forestales del Montseny manifiestan una elevada capacidad de neutralización de las precipitaciones ácidas, puesto que el $\mathrm{pH}$ medio de 5 arroyos de cuencas silicatadas muestreados regularmente fue de 6.6-7.8 (Rodà 1983). Las tierras pardas del Montseny están seguramente en el rango de tamponamiento de los silicatos (Ulrich 1983), y la neutralización debe conseguirse fundamentalmente por la meteorización química de los aluminosilicatos del suelo, que consume un equivalente de $\mathrm{H}^{+}$por cada equivalente de cationes metálicos liberado a la solución del suelo (Krauskopf 1979). La capacidad de neutralización debida a la meteorización de los silicatos puede ser de 0.2-2 $\mathrm{keq} \mathrm{ha}^{-1} \mathrm{año}^{-1}$ (Ulrich 1983). La deposición actual de ácido en el Montseny está en el límite inferior de este intervalo (0.13-0.29 $\mathrm{keq} \mathrm{ha}^{-1}$ año ${ }^{-1}$; tabla 1). Esta forma de neutralización podría no ser perjudicial para el ecosistema, ya que las reservas de minerales del suelo permitirían una celebración moderada de la meteorización sin merma 
sensible. Sin embargo, otra vía de neutralización es el intercambio catiónico en el suelo, con sustitución de los cationes metálicos (principalmente $\mathrm{Ca}^{2+}, \mathrm{Mg}^{2+}, \mathrm{y} \mathrm{K}^{+}$), adsorbidos en la superficie de las arcillas y de los coloides orgánicos, por iones $\mathrm{H}^{+}$. Este intercambio catiónico podría traducirse a largo plazo en una acidificación del suelo y en un empobrecimiento del perfil en nutrientes al lixiviarse los cationes desplazados.

\section{Trascolación}

La trascolación (throughfall) es la precipitación que puede recogerse con pluviómetros situados dentro del bosque. No incluye la escorrentía cortical (stemflow), que es el agua que resbala por los troncos.

En los 3 bosques estudiados, la trascolación es en promedio menos ácida que la deposición global (tabla 1). Sólo el 35\% del flujo anual de hidrogeniones libres en la deposición global del encinar llega al suelo del bosque con la trascolación. En el hayedo y el abetal, las cifras son el $45 \%$ y el $63 \%$, respectivamente. En términos absolutos, la mayor neutralización se produce en el hayedo, seguido del abetal y del encinar, con 14.4, 10.6 , y 8.4 meq $^{2}$ año-1, respectivamente, de $\mathrm{H}^{+}$retenidos o neutralizados por las copas. Esta neutralización parcial de las precipitaciones ácidas puede producirse por: (1) el lavado de deposición seca neutralizante; (2) el intercambio catiónico en las superficies vegetales y en los espacios intercelulares de las hojas (Mecklenburg et al. 1966); y (3) el lavado de sales de ácidos débiles, cuyos aniones se protonarían en medio ácido (Hoffman et al. 1980).

La trascolación neta (net throughfall) es el resultado de restar los aportes en la deposición global a los aportes en la trascolación, y representa las cantidades de nutrientes lavadas de las copas (prescindiendo de la escorrentía cortical). Los nutrientes de la trascolación neta proceden de la deposición seca (aerosoles impactados y gases absorbidos) acumulada sobre las copas, y de la lixiviación de los árboles y de sus epibiontes. Es decir, la trascolación neta incluye nutrientes que entran en el ecosistema (deposición seca) y nutrientes que, habiendo sido previamente absorbidos por las raíces, circulan internamente por el ecosistema.

Todos los aportes netos en la trascolación han resultado positivos en este estudio, excepto los de $\mathrm{H}^{+}$, que son negativos en los 3 bosques (tabla 1). 
El encinar se caracteriza por tener aportes netos relativamente altos de $\mathrm{PO}_{4}^{3-}$, relativamente bajos de $\mathrm{Ca}^{2+}$, y muy bajos de $\mathrm{Na}^{+}$y $\mathrm{NO}_{3}^{-}$ (tabla 1). En el encinar, y en términos de equivalentes, se lava más $\mathrm{K}^{+}$ que $\mathrm{Ca}^{2+}$, mientras que en el hayedo y el abetal sucede lo contario. El encinar montano de La Castanya tiene unos aportes netos de $\mathrm{K}^{+}$y $\mathrm{Mg}^{2+}$ en la trascolación muy parecidos a los del encinar de Rouquet (Rapp 1970), pero los aportes netos de $\mathrm{Ca}^{2+}$, y quizá los de $\mathrm{Na}^{+}$, son mayores en este último, que se encuentra sobre sustrato calcáreo.

El hayedo de Santa Fe no destaca especialmente de los otros hayedos europeos por sus aportes netos en la trascolación. En particular, los datos disponibles no revelan la existencia de un gradiente latitudinal en la lixiviación de $\mathrm{K}^{+}$. En el conjunto de hayedos estudiados, el de Santa Fe se sitúa en la gama alta de los aportes netos de $\mathrm{NO}_{3}^{-}$; en la intermedia para los de $\mathrm{K}^{+}, \mathrm{Ca}^{2+}$, y $\mathrm{PO}_{4}^{3-}$; y en la baja para los de $\mathrm{Na}^{+}$y $\mathrm{Mg}^{2+}$.

El abetal se diferencia claramente del hayedo y del encinar porque sus aportes netos son muy elevados para todos los iones (excepto $\mathrm{PO}_{4}^{3-}$; tabla 1). Las proporciones iónicas en los aportes netos del abetal son sin embargo muy parecidas a las del hayedo. Es decir, la precipitación lava mucho más nutrientes de las copas de los abetos que de las hayas, pero lo hace en las mismas proporciones para los distintos iones analizados. Matzner et al. (1982) en Alemania y Nihlgard (1970) en Suecia encontraron también que la trascolación de plantaciones de Picea abies contenía mucho más nutrientes que la de hayedos adyacentes. No obstante, la diferencia encontrada en el Montseny es en general bastante mayor que en estos dos estudios. Los aportes netos de $\mathrm{K}^{+}, \mathrm{Ca}^{2+}, \mathrm{y}$ $\mathrm{NO}_{3}^{-}$en el abetal del Montseny figuran entre los más elevados de la bibliografia sobre bosques templados. Es posible que hayamos sobreestimado muestralmente los aportes netos del abetal, porque su variabilidad espacial es muy alta y utilizamos sólo 5 colectores de trascolación en este bosque. Sin embargo, tanto la deposición seca como la lixiviación pueden ser elevadas en el abetal, debido a que: (1) la longitud de las copas es considerablemente mayor que en el hayedo y en el encinar; (2) el techo del bosque es más irregular (mayor canopy roughness); (3) el área total de las hojas y ramas es mayor; y (4) la hojas aciculares capturan aerosoles más eficazmente que las hojas planas (Woodcock 1953). Es interesante señalar que, a diferencia de lo que sucede en Europa, los abetales americanos de $A$. balsamea (Olson et al. 1981) y de $A$. grandis (Tiedemann et al. 1980) dan lugar a una trascolación 
pobre en nutrientes. Los aportes netos bajo $A$. balsamea son incluso inferiores a los de un bosque caducifolio de la misma región (Eaton et al. 1973, Olson et al. 1981). Esta diferencia del comportamiento de coníferas y caducifolios entre Europa y Norteamérica podría deberse a diferencias entre las especies implicadas, o a que las concentraciones de gases y aerosoles fuesen mayores en las áreas de estudio europeas, permitiendo una mayor deposición seca.

La mayor parte del $\mathrm{K}^{+}$que se lava de las copas de los árboles procede de la lixiviación, ya que los aportes atmosféricos de este ión son generalmente pequeños y, en cambio, es un nutriente abundante, muy móvil, y fácilmente lixiviable en los tejidos vegetales. En el encinar montano, una parte importante del lavado de $\mathrm{Mg}^{2+}$ y $\mathrm{PO}_{4}^{3-}$ parece también proceder de lixiviación porque, al igual que $\mathrm{el}^{+}$, sus aportes netos presentan un máximo durante el período de senescencia foliar y de floración de la encina (junio-julio, en La Castanya), y porque están positivamente correlacionados con la cantidad de trascolación, con la intensidad del color orgánico, y con los aportes netos de $\mathrm{K}^{+}$. En el hayedo y el abetal es difícil evaluar la importancia relativa de la deposición seca y de la lixiviación en el lavado de nutrientes de las copas.

\section{Desfronde}

Se denomina desfronde o caída de hojarasca (litterfall) al conjunto de órganos vegetales (hojas, ramas, frutos, inflorescencias, cortezas, etc.). y de restos animales que caen al suelo del bosque procedentes de los distintos estratos de la vegetación. El desfronde constituye, junto a la mortalidad de las raíces, la principal vía de entrada de materia orgánica al suelo del ecosistema. Los nutrientes contenidos en la hojarasca son liberados durante el proceso de descomposición, pudiendo ser absorbidos de nuevo por la vegetación. La cantidad y características de los materiales del desfronde determinan en buena medida las pautas de circulación de nutrientes en los ecosistemas forestales.

El desfronde anual de los 3 bosques estudiados se presenta en la tabla 2. El valor más alto de desfronde total se registró en el encinar montano, y el más bajo en el abetal. Los 3 bosques difieren en cuanto a variabilidad interanual: en el hayedo, el desfronde fue muy similar en los 2 años de muestreo, no existiendo diferencia significativa entre ambos 


\section{Tabla 2.}
Desfronde anual en tres ecosistemas forestales del Montseny (Barcelona)

\begin{tabular}{|c|c|c|c|}
\hline fracción & encinar & hayedo & abetal \\
\hline & \multicolumn{3}{|c|}{$\mathrm{t} h \mathrm{~h}^{-1} \mathrm{año}^{-1}$} \\
\hline hojas & 3.06 & 3.23 & 2.31 \\
\hline $\operatorname{ramas}_{\text {finas }}{ }^{1}$ & 0.92 & 0.22 & 0.40 \\
\hline ramas gruesas $^{2}$ & 0.45 & 0.16 & 0.15 \\
\hline frutos & 0.58 & 0.36 & 0.41 \\
\hline resto & 0.30 & 0.34 & 0.17 \\
\hline Total & 5.31 & 4.30 & 3.43 \\
\hline
\end{tabular}

( $p>0.05$ ); en cambio, en el encinar ( 3 años de datos) y en el abetal ( 2 años) las variaciones interanuales fueron más importantes y alcanzaron la significción estadística $(\mathrm{P}<0.05)$.

En los bosques del Montseny, las proporciones de las distintas fracciones del desfronde siguen la pauta general observada en los ecosistemas forestales (Bray \& Gorham 1964). Las hojas de la especie arbórea dominante constituyen la fracción principal: $75 \%$ del peso seco total en el hayedo, $67 \%$ en el abetal, y $58 \%$ en el encinar. Siguen en importancia las ramas (finas y gruesas), siendo particularmente destacable su participación en el encinar ( $27 \%$ del desfronde total). En los 3 bosques, la contribución de hojas y ramas es del $83 \%$ del total. La caída de frutos representa un 8-12\% del desfronde anual. Kira \& Shidei (1967) consideran que, en los bosques maduros, el desfronde foliar tiende al $50 \%$ del total. Como hemos visto, en los bosques del Montseny es el encinar el que presenta un menor porcentaje de la fracción hojas, aunque sea el abetal el más maduro desde el punto de vista de la edad de los árboles. Cabe seña- 
lar al respecto que en el abetal se subestimó seguramente la fracción gruesa del desfronde, debido a que repetidos desperfectos en las trampas de muestreo impidieron la recogida de esta fracción en algunos períodos. Por otra parte, los abetos retienen las ramas muertas durante largo tiempo, y la biomasa de ramas muertas en pie es mucho mayor en el abetal que en los otros dos bosques. Esto contribuiría a que el porcentaje de hojas en el desfronde se mantuviese elevado incluso en la etapa de madurez del bosque.

La variación estacional del desfronde es distinta en cada uno de los 3 bosques estudiados (tabla 3 ). En el hayedo, la estación predominante de caída es el otoño, como acostumbra a suceder en los bosques caducifolios. En el encinar, acorde con su carácter mediterráneo, la caída más importante se produce a finales de primavera y durante el verano, coincidiendo con la estación seca. En el abetal, verano y otoño comparten la primacía.

\section{Aportes de nutrientes por el desfronde}

En la tabla 4 se representan las concentraciones medias de nutrientes en las hojas del desfronde de las 3 especies arbóreas. Las hojas de haya

\section{Tabla 3.}

\section{Variación estacional del desfronde en tres ecosistemas forestales del Montseny (Barcelona)}

Se dan los porcentajes respecto al desfronde total anual

\begin{tabular}{lrrr}
\hline estación & encinar & hayedo & abetal \\
\hline & 15.1 & 5.3 & 18.4 \\
diciembre-febrero & 16.9 & 6.2 & 5.2 \\
marzo-mayo & 48.4 & 3.6 & 38.7 \\
junio-agosto & 19.6 & 84.9 & 37.7 \\
septiembre-noviembre & & & \\
Total & 100.0 & 100.0 & 100.0
\end{tabular}


son las más concentradas en $\mathrm{K}$ y $\mathrm{Na}$ (y, por escaso margen, en $\mathrm{Mg}$ ), que son los elementos más móviles dentro de la planta. Las hojas de abeto son las que tienen una mayor concentración de $\mathrm{Ca}$, elemento estructural por excelencia. Las hojas de encina son las de mayor contenido en $\mathrm{N}$ y $\mathrm{P}$, que probablemente sean los nutrientes de mayor importancia para el ecosistema. Sin embargo, la concentración de $\mathrm{N}$ en las hojas del desfronde de encina es sólo un 5\% mayor que en las de haya, y un 13\% mayor que en las de abeto (tabla 4).

En la tabla 5 pueden verse los aportes anuales de nutrientes por el desfronde en los 3 bosques. El encinar montano es el que tiene mayor circulación de $\mathrm{N}, \mathrm{P}, \mathrm{Ca}, \mathrm{y} \mathrm{Mg}$ en el desfronde. Los aportes de $\mathrm{K}$ y $\mathrm{Na}$ son sensiblemente iguales en el encinar y en el hayedo. Excepto en $\mathrm{P}$ y $\mathrm{Ca}$, los aportes del abetal son bastante inferiores a los del hayedo. Con las excepciones indicadas, la ordenanción de los 3 bosques según sus aportes por el desfronde es la misma que por la cantidad de caída de hojarasca: encinar $>$ hayedo $>$ abetal (tabla 5).

Los aportes de $\mathrm{N}$ por el desfronde son prácticamente iguales en el encinar montano de La Castanya que en el encinar de Rouquet ( 35 v. 34

\section{Tabla 4.}

Concentraciones medias ponderadas de nutrientes en las hojas recién caídas de encina, haya, y abeto del Montseny (Barcelona)

\begin{tabular}{llllllllll}
\hline Especie & $\begin{array}{c}\text { Desfronde } \\
\text { foliar }\end{array}$ & $\mathrm{N}$ & $\mathrm{P}$ & $\mathrm{K}$ & $\mathrm{Ca}$ & $\mathrm{Mg}$ & $\mathrm{Na}$ \\
\hline
\end{tabular}

$\mathrm{tha}^{-1}$

3.06

6.14

0.83

4.51

8.33

1.15

5.75

9.83

1.21

0.12

Haya

3.23

5.84

0.40

5.42

0.63

13.67

1.06

0.16

Abeto

2.31

5.20

13.67

0.12 


\section{Tabla 5.}

Aportes de nutrientes por el desfronde en tres ecosistemas forestales del Montseny (Barcelona)

Se incluyen la fracción fina y la fracción gruesa.

\begin{tabular}{lcccccccc}
\hline Bosque & $\begin{array}{c}\text { Desfronde } \\
\text { foliar }\end{array}$ & $\mathrm{N}$ & $\mathrm{P}$ & $\mathrm{K}$ & $\mathrm{Ca}$ & $\mathrm{Mg}$ & $\mathrm{Na}$ \\
\hline & & & & & & & \\
& $\mathrm{t} \mathrm{ha}^{-1} \mathrm{año}^{-1}$ & & & & $\mathrm{~kg} \mathrm{ha}^{-1}$ & $\mathrm{año}^{-1}$ & & \\
\cline { 6 - 8 } & & & & & & & & \\
Encinar & 5.31 & 35.0 & 4.26 & 21.9 & 47.8 & 5.86 & 0.53 \\
Hayedo & 4.30 & 25.3 & 1.78 & 22.6 & 38.0 & 4.47 & 0.57 \\
Abetal & 3.43 & 21.9 & 2.26 & 15.9 & 37.3 & 3.10 & 0.34 \\
\hline
\end{tabular}

$\mathrm{kg} \mathrm{ha}^{-1}$ año ${ }^{-1}$; Rapp 1970). Los aportes de $\mathrm{P}$ son en cambio un $48 \%$ mayores en La Castanya, los de $\mathrm{K}$ un $33 \%$ mayores, y los de $\mathrm{Mg}$ un $26 \%$ mayores. Los aportes de Ca son un $37 \%$ mayores en Rouquet, que está sobre sustrato calcáreo. Utilizando la media de 4 años en Rouquet, período durante el cual se midieron los flujos de nutrientes (Rapp 1970), el desfronde total resulta ser un $38 \%$ mayor en La Castanya; si utilizamos la media de 7 años en Rouquet (Lossaint \& Rapp 1978), el desfronde es un $26 \%$ mayor en La Castanya.

Hemos comparado los aportes de nutrientes por el desfronde en el hayedo de Santa Fe con la media registrada en 10 hayedos europeos (Astrup \& Bülow-Olsen 1979, Cole \& Rapp 1981, Lemée 1978, Nihlgård y Lindgren 1977). Los aportes de $\mathrm{N}$ en el hayedo del Montseny representan tan sólo el $49 \%$ del valor de dicha media ( $25.3 \mathrm{v} .51 .5 \mathrm{~kg} \mathrm{ha}^{-1}$ año $\left.{ }^{-1}\right)$, y los de $\mathrm{P}$ representan el 63\% . Los aportes de K, Ca, y Mg en el hayedo de Santa Fe son sensiblemente iguales a la media europea. Entre las localidades europeas estudiadas, la que más se parece a nuestro hayedo por sus aportes de $\mathrm{N}$ es la de Langarod (Suecia; Nihlgård \& Lindgren 1977), con $31 \mathrm{~kg} \mathrm{ha}^{-1}$ año ${ }^{-1}$, sobre suelo oligotrófico. Señalemos sin embargo 
que Nihlgård \& Lindgren no encontraron una relación clara entre el grado trófico del bosque y los aportes de $\mathrm{N}$ por el desfronde en los 3 hayedos que estudiaron. El desfronde total (peso seco) del hayedo de Santa Fe es un $17 \%$ superior al de la media europea. Esto quiere decir que el hayedo de nuestra parcela experimental produce la misma o mayor cantidad de hojarasca con un flujo asociado de $\mathrm{N}$ mucho menor que la media de los otros hayedos europeos. Si consideramos, como hace Vitousek (1982), que la cantidad de materia seca producida en el desfronde por unidad de nutriente contenida en el desfronde (es decir, el inverso de la concentración del nutriente) es una medida de la eficiencia con la cual el

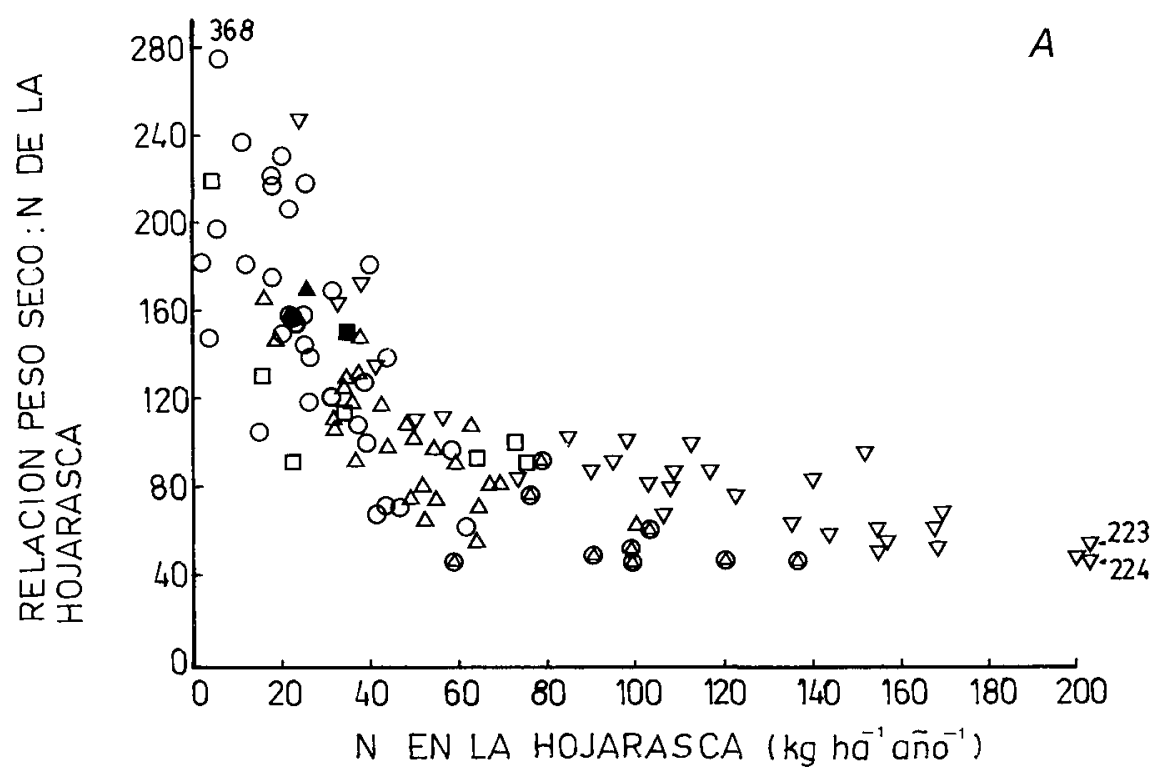

Figura 1. Relación entre los aportes de (a) N, (b) P, y (c) Ca por el desfronde (abcisas) y la eficiencia en el uso de cada nutriente expresada como peso seco de desfronde por unidad de peso del nutriente retornada en el desfronde (ordenadas). Este gráfico es una reproducción del que da Vitousek (1982), añadiéndole los tres bosques estudiados en el Montseny.

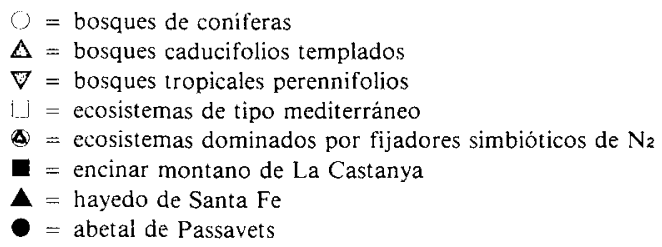



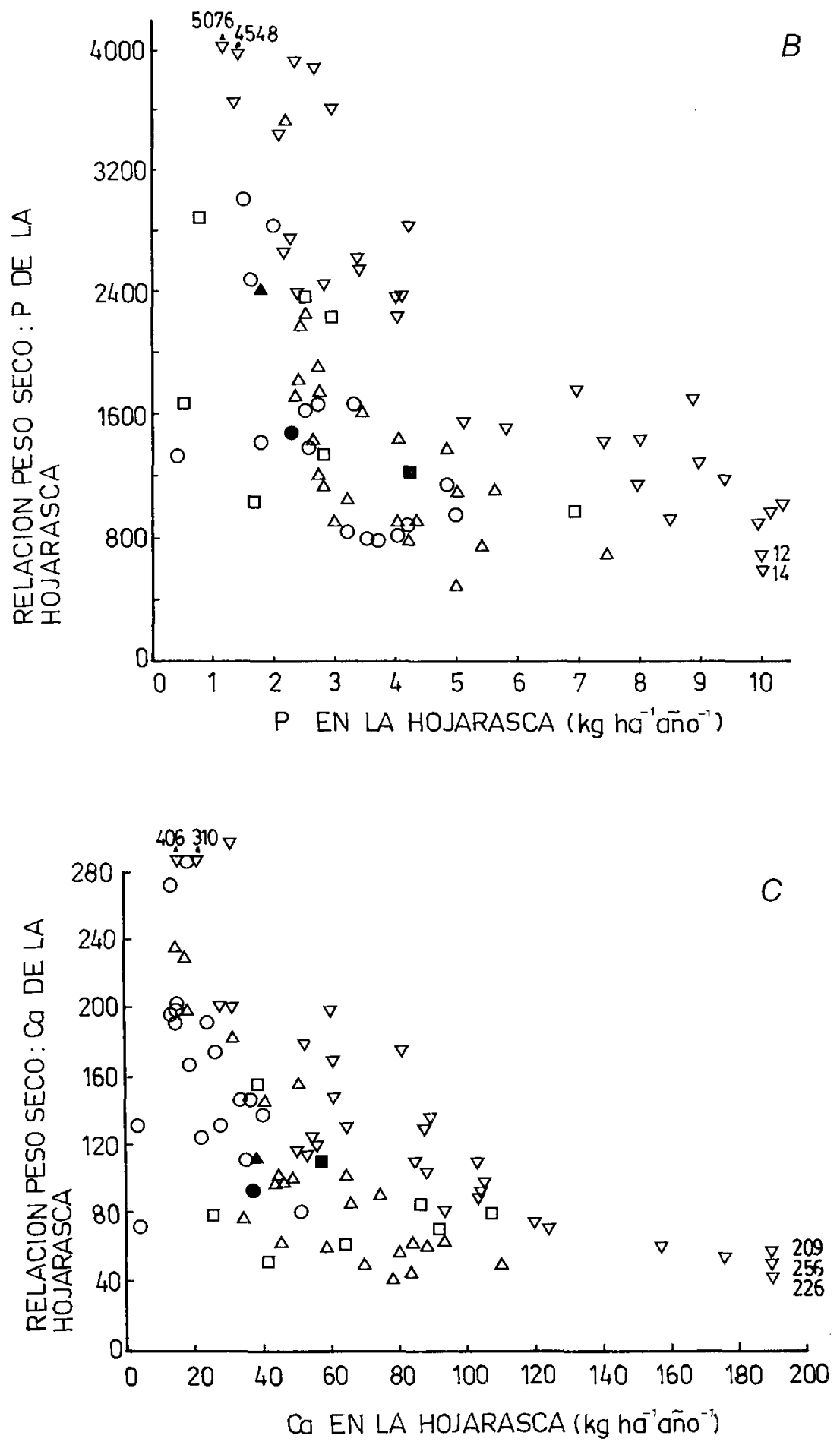
bosque utiliza dicho nutriente, el hayedo de Santa Fe utiliza más eficazmente el $\mathrm{N}$ que la media de los hayedos europeos. Es posible que esto sea debido a una menor disponibilidad de $\mathrm{N}$ en el suelo, originada tal vez por el retardo de la descomposición a que da lugar la falta recurrente de agua.

Utilizando el criterio anterior, en la Figura 1 se represénta la eficiencia en el uso de 3 nutrientes $(\mathrm{Ca}, \mathrm{N}, \mathrm{y} \mathrm{P})$ respecto a sus aportes por el desfronde. Hemos elegido estos 3 elementos porque su retorno al suelo del bosque se realiza fundamentalmente con el desfronde (tablas 1 y 5 ; Cole \& Rapp 1981). La eficiencia en el uso del $N$ es similar en los 3 bosques estudiados (Figura 1), siendo el hayedo algo más eficaz que el abetal, y éste algo más que el encinar. En la Figura 1 se aprecia claramente lo que antes comentábamos: el hayedo de Santa Fe utiliza el N mucho más eficazmente que la mayor parte de los bosques caducifolios templados. La eficiencia del abetal para el $\mathrm{N}$ es parecida a la media de los bosques de coníferas, mientras que la del encinar es superior a la media de los ecosistemas de tipo mediterráneo.

En el caso del P, el hayedo es mucho más eficaz que el abetal y el encinar, y que todos los bosques caducifolios templados de los que se dispone de datos (excepto uno). El encinar de La Castanya figura entre los ecosistemas de tipo mediterráneo menos eficaces en el uso del P. Para el $\mathrm{Ca}$, las eficiencias de los 3 bosques estudiados difieren poco entre sí. El abetal de Passavets es uno de los bosques de coníferas de menor eficiencia en el uso del $\mathrm{Ca}$.

La imagen general que se obbtiene de la Figura 1, junto a los datos de concentraciones y aportes en el desfronde (tablas 4 y 5), es que el encinar montano de La Castanya funciona con una economía holgada en $\mathrm{P}$, y suficiente o moderadamente escasa en N. El hayedo de Santa Fe, que es considerado eutrófico en el contexto del Montsény (Bolòs 1983), parece funcionar en base a una economía deficitaria tanto en $\mathrm{N}$ como en $\mathrm{P}$. El abetal de Passavets manifiesta también una economía frugal de $\mathrm{N}$, como sucede en general en las coníferas (Gosz 1981), pero utiliza el Ca con mayor prodigalidad de la habitual en otras gimnospermas.

\section{Biomasa, producción y mineralomasa en el encinar montano}

En el encinar montano de La Castanya hemos estudiado la bioma- 

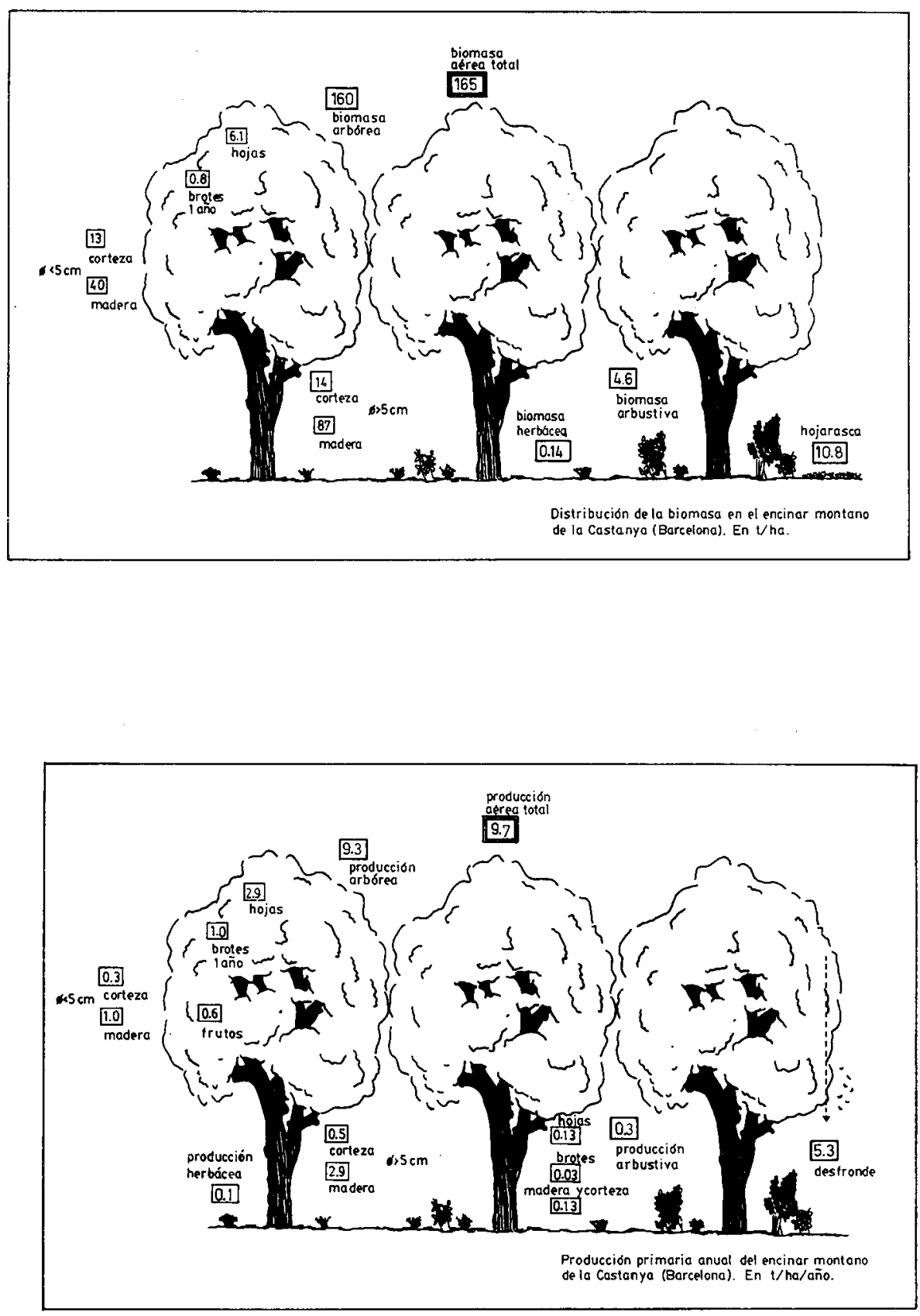


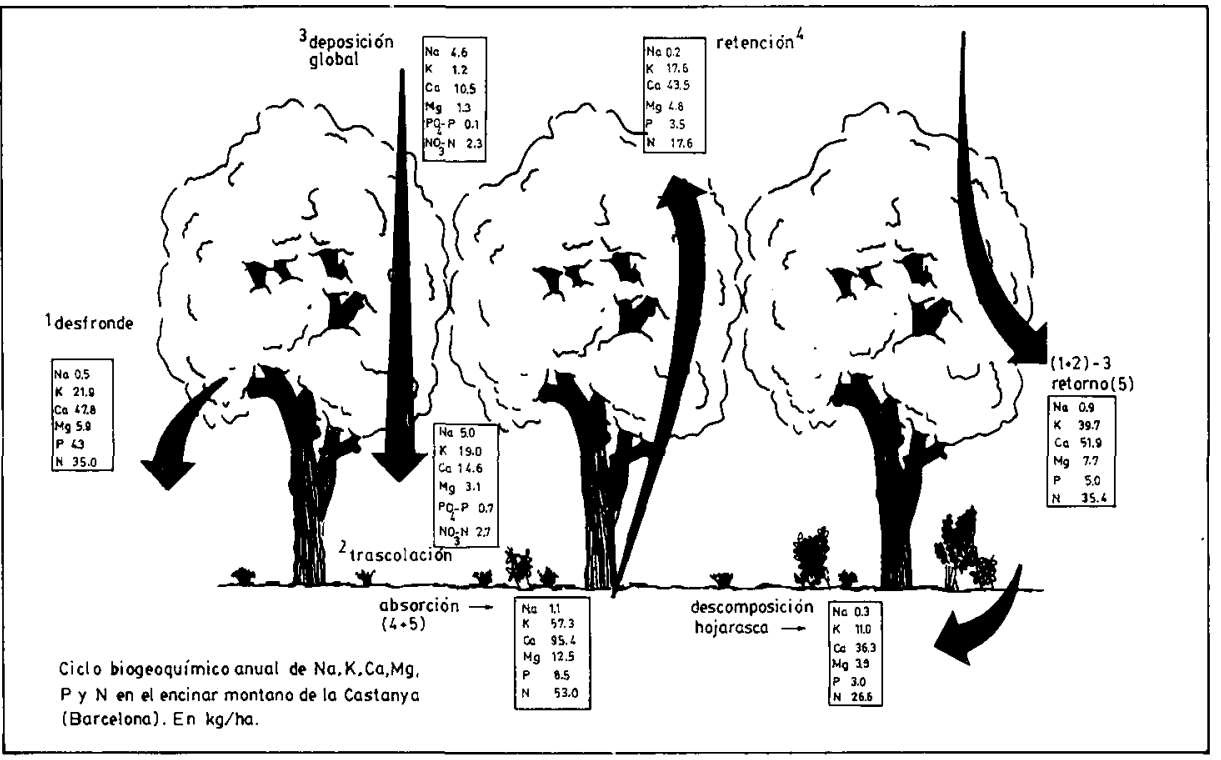

Tabla 6.

Mineralomasas de seis nutrientes en el encinar montano de la Castanya (Montseny, Barcelona)

\begin{tabular}{lrrrrrr}
\hline Estrato y fracción & $\mathrm{N}$ & $\mathrm{P}$ & $\mathrm{K}$ & $\mathrm{Ca}$ & $\mathrm{Mg}$ & $\mathrm{Na}$ \\
\hline & & & & & & \\
ESTRATO ARBOREO & & & $\mathrm{kg}$ & $\mathrm{ha}^{-1}$ & & \\
\cline { 2 - 7 } & & & & & & \\
Hojas & 5.9 & 1.6 & 29.4 & 41.0 & 8.5 & 0.40 \\
Brotes del año & 87.2 & 35.9 & 101.8 & 146.3 & 20.6 & 1.27 \\
Madera $\varnothing<5 \mathrm{~cm}$ & 108.4 & 29.5 & 158.7 & 215.9 & 38.2 & 1.30 \\
Madera $\varnothing>5 \mathrm{~cm}$ & 62.8 & 4.8 & 39.1 & 347.7 & 14.2 & 0.67 \\
Corteza $\varnothing<5 \mathrm{~cm}$ & 63.8 & 4.3 & 50.1 & 438.8 & 24.0 & 0.50 \\
Corteza $\varnothing>5 \mathrm{~cm}$ & 410.7 & 83.1 & 384.3 & 1195.8 & 106.9 & 4.18 \\
Total arbóreo & 17.0 & 3.5 & 12.9 & 39.5 & 3.2 & 0.18 \\
& 1.5 & 0.2 & 1.9 & 1.2 & 0.3 & 0.02 \\
ESTRATO ARBUSTIVO & & & & & & \\
ESTRATO HERBACEO & & & & & & \\
TOTAL AEREO & 429.2 & 86.8 & 399.0 & 1236.5 & 110.4 & 4.38 \\
\hline
\end{tabular}


sa, la producción, la mineralomasa (nutrientes contenidos en la biomasa), y la inmovilización anual de nutrientes en la producción leñosa. Los resultados están en las Figuras 2 y 3, y en la tabla 6.

La biomasa área de la parcerla experimental es de $165 \mathrm{t} \mathrm{ha}^{-1}$ (peso seco), de las cuales 160 corresponden al estrato arbóreo, repartidas en $6.1 \mathrm{t} \mathrm{ha}^{-1}$ de hojas ( $3.8 \%$ de la biomasa arbórea), y $154 \mathrm{t} \mathrm{ha} \mathrm{a}^{-1}$ de madera y corteza. La biomasa de hojas es muy parecida a la de otros encinares (Lossaint \& Rapp 1978, Susmelt et al. 1976), y superior a los valores habituales en los bosques caducifolios (1-5 $\mathrm{t} \mathrm{ha}^{-1}$; Gosz 1981).

La producción aérea total del encinar de La Castanya es de $9.7 \mathrm{t}$ $\mathrm{ha}^{-1} \mathrm{año}^{-1}$ (peso seco), de las cuales 9.3 corresponden al estrato arbóreo, repartidas en $3.1 \mathrm{t} \mathrm{ha}^{-1}$ año ${ }^{-1}$ de hojas, 0.6 de frutos, y 5.6 de madera y corteza (incluyendo los brotes del año). Esta producción total es elevada para un encinar, pero parece congruente con la situación de la parcela experimental, que tiene una pendiente escasa y un suelo relativamente profundo; incluso en épocas de sequía no se ha observado un stress hídrico acusado en las encinas de la parcela (Rabella et al. 1983). Si a la producción leñosa aérea de los árboles de la parcela $\left(5.6 \mathrm{t}^{-1}\right.$ año-1 $)$ le restamos el desfronde leñoso (1.4 $\mathrm{t} \mathrm{ha}^{-1} \mathrm{año}^{-1}$; tabla 2 ) obtenemos un incremento de la biomasa leñosa arbórea aérea de $4.2 \mathrm{t} \mathrm{ha}^{-1} \mathrm{año}^{-1}$. El tiempo de duplicación correspondiente a esta biomasa $\left(154 \mathrm{tha}^{-1}\right)$ sería de 37 años, a incremento constante.

Las mineralomasas del encinar de La Castanya (tabla 6) son relativamente altas para un bosque cuya biomasa es sólo moderada. Destaca en particular el $\mathrm{Ca}$, con una mineralomasa aérea de $1237 \mathrm{~kg} \mathrm{ha}^{-1}$, seguido del $\mathrm{N}$ con $429 \mathrm{~kg} \mathrm{ha}^{-1} \mathrm{y}$ del $\mathrm{K}$ con $399 \mathrm{~kg} \mathrm{ha}^{-1}$. Como es habitual en los ecosistemas forestales, la madera de troncos y ramas contiene la mayor parte de los nutrientes almacenados en los árboles, debido al predominio de la biomasa de esta fracción (Figura 2). En el encinar de La Castanya la mayor parte del Ca se acumula sin embargo en las cortezas, con un total de $786.5 \mathrm{~kg} \mathrm{Ca} \mathrm{ha}^{-1}$. La mineralomasa foliar representa los

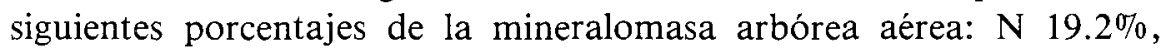
$\mathrm{P} 8.8 \%, \mathrm{~K} 7.4 \%$, Ca $3.3 \%, \mathrm{Mg} 7.7 \%$, y $\mathrm{Na} 9.1 \%$. Teniendo en cuenta que la biomasa foliar es sólo el $3.8 \%$ de la total, las cifras anteriores ponen de manifiesto la riqueza de las hojas de todos los nutrientes (excepto Ca). Destaca especialmente la importancia del compartimento foliar en el caso del $\mathrm{N}$. 
Si comparamos las concentraciones de nutrientes en las hojas de encina (este estudio, Lossaint \& Rapp 1978, Leonardi \& Rapp 1980, Terradas \& Escarré 1983) con las encontradas en hojas de coniferas y cadufifolios (Cole $\&$ Rapp 1981) vemos que las encinas tienen una composición más parecida a la de las coníferas, ya que los caducifolios presentan concentraciones claramente superiores de $\mathrm{N}$ y K. Considerando las concentraciones de los elementos en el total de la biomasa y comparándolas con las de bosques caducifolios de la zona templada y de las comunidades arbustivas de tipo mediterráneo (Cole \& Rapp 1981, Gray \& Schlesinger 1981), los encinares tienen concentraciones superiores a las de los bosques caducifolios para todos los nutrientes examinados, debido a que la madera de encina es ligeramente más rica en nutrientes. Las comunidades de arbustos mediterráneos (Gray \& Schlesinger 1981, Lossain \& Rapp 1971) presentan concentraciones aún mayores, debido principalmente a la mayor importancia relativa de la biomasa foliar.

El alto contenido de nutrientes en la madera de encina, junto a los almacenados en las hojas de años anteriores, pueden representar importantes reservas movilizables, lo cual independizaría hasta cierto punto a los árboles frente a las variaciones en la disponibilidad de nutrientes en el suelo. Esto podría constituir una adaptación de las encinas a medios fluctuantes donde la disponibilidad de nutrientes durante la estación cálida puede verse reducida por la falta de agua. Esta estrategia en el uso de los nutrientes confiere ventajas a los vegetales perennifolios en medios oligotróficos, como ha sido puesto de manifiesto por Gray (1983) al comparar las eficiencias en el uso de nutrientes por parte de arbustos perennifolios y caducifolios de California.

\section{Ciclos biogeoquímicos en el encinar montano}

El conocimiento de las mineralomasas y de la inmovilización anual en la producción del encinar, junto a los flujos en la deposición global, la trascolación, y el desfronde, permite una visión mucho más completa de los ciclos de nutrientes en este bosque que en el hayedo o el abetal. La información de que disponemos sobre los flujos anuales de nutrientes en el encinar montano se resume en la Figura 4. Señalemos que hay lagunas importantes en este esquema, como pueden ser la fijación biológica de $\mathrm{N}_{2}$, los flujos en la escorrentía cortical y en el material microparticulado del desfronde, los flujos de $\mathrm{NH}_{4}$, de $\mathrm{N}$ orgánico, y de $\mathrm{P}$ orgánico en las aguas de lluvia, y la incapacidad de distinguir cuantitativamente la depo- 
sición seca de la lixiviación. No obstante, los datos de la Figura 6 permiten hacer algunas consideraciones que no pueden percibirse examinando los flujos individuales.

El retorno de nutrientes al suelo (desfronde + trascolación neta) expresa las cantidades de los distintos elementos que circulan con un ciclo rápido interno al ecosistema. La retención está formada por los nutrientes que se incorporan a la biomasa de troncos y ramas que se produce cada año, y que entran por lo tanto en un ciclo mucho más lento. La suma del retorno y de la retención es la absorción (uptake; Cole \& Rapp 1981). En rigor, al calcular la absorción anual no debiera contabilizarse en el retorno la fracción leñosa del desfronde, pero es práctica habitual utilizar el retorno total. Los requerimientos (requirement; Cole \& Rapp 1981) son la suma de la retención y de la mineralomasa de las hojas producidas en el año en curso. Cuando los requerimientos son mayores que la absorción, la planta debe cubrir el resto de sus necesidades de nutrientes retranslocando nutrientes de los órganos viejos a los órganos nuevos. Cole (1981) utiliza de hecho la diferencia entre requerimientos y absorción con una estima de la magnitud de la retranslocación. Aplicando este criterio al encinar de La Castanya, sólo parece haber retranslocación en el caso del $\mathrm{N}$, y resulta ser de $7 \mathrm{~kg} \mathrm{ha}^{-1} \mathrm{año}^{-1}(11 \%$ de los requerimientos). Cole (1981) encontró que los bosques caducifolios del IBP Woodland Data Set retranslocaban en promedio $24 \mathrm{~kg} \mathrm{~N} \mathrm{ha}^{-1}$ año ${ }^{-1}$ ( $25 \%$ de sus requerimientos), mientras que las coníferas no tenían, en promedio, retranslocación aparente. El encinar montano ocuparía pues una posición intermedia entre coníferas y caducifolios.

Otra forma de estimar la retranslocación consiste en comparar las concentraciones medias de las hojas del desfronde con las de las hojas verdes de 2 ó más años de edad. Las concentraciones de $\mathrm{P}, \mathrm{K}$; y Mg son menores en las hojas del desfronde que en las hojas verdes, pero parte de esta diferencia podría ser debida a la lixiviación, ya que para los 3 nutrientes se produce un aumento de los aportes netos por trascolación en la época de senescencia. En el caso de $\mathrm{Ca}$ y $\mathrm{Na}$, las concentraciones en las hojas del desfronde son iguales o mayores que en las hojas verdes. En cambio, la concentración de $\mathrm{N}$ en las hojas del desfronde es sólo la mitad de la existente en las hojas verdes de 2 ó más años, lo cual corresponde a la desaparición de unos $22 \mathrm{~kg} \mathrm{ha}^{-1} \mathrm{año}^{-1}$ antes de la caída. Como la lixiviación de $\mathrm{N}$ total en los ecosistemas forestales difícilmente excede de 10 $\mathrm{kg} \mathrm{ha}^{-1} \mathrm{año}^{-1}$ (y, en general, es mucho menor que dicha cifra, cf. Parker 
1983), se deduce que la mayor parte del $\mathrm{N}$ que desaparece de las hojas antes de su caída debe ser retranslocado. Pueden darse también pérdidas de $\mathrm{N}$ en las hojas senescentes debido a la volatilización de amoníaco (Farquhar et al. 1979). Problamente la discrepancia entre las dos estimas de la retranslocación de $\mathrm{N}\left(7 \mathrm{v} .22 \mathrm{~kg} \mathrm{ha}^{-1} \mathrm{año}^{-1}\right)$ se reduciría apreciablemente al incorporar la lixiviación y la volatilización. La discrepancia residual podría ser debida a los errores experimentales (por ejemplo, la estima de $7 \mathrm{~kg} \mathrm{ha}^{-1}$ año ${ }^{-1}$ se obtiene como una diferencia pequeña entre dos cantidades de mayor magnitud), o podría indicar que el $\mathrm{N}$ en el encinar se retransloca fundamentalmente de las hojas viejas a los frutos. Esta vía de retranslocación está contemplada en la estima de $22 \mathrm{~kg} \mathrm{ha}^{-1} \mathrm{año}^{-1}$, pero no en la otra, porque el $\mathrm{N}$ de los frutos se contabiliza en el retorno $y$, por tanto, no aparece como retranslocado al restar la absorción de los requerimientos. $\mathrm{El}$ paso del $\mathrm{N}$ de las hojas viejas a los frutos se facilita en el encinar porque la senescencia foliar coincide con el inicio del desarrollo de los frutos.

Igual que hacíamos para el desfronde, podemos calcular la eficiencia en el uso del $\mathrm{N}$ por parte del encinar, dividiendo la producción primaria neta por la absorción, los requerimientos, o el retorno de N. Obtenemos así los kilos de materia seca producidos por cada kilo de $\mathrm{N}$ utilizado. Los resultados para el encinar montano son:

$\begin{array}{llll}\text { producción/absorción } & =182 \mathrm{~kg} \text { peso seco } / \mathrm{kg} \mathrm{N} \\ \text { producción/requerimientos } & =162 \mathrm{~kg} \text { peso seco } / \mathrm{kg} \mathrm{N} \\ \text { producción/retorno } & =273 \mathrm{~kg} \text { peso seco } / \mathrm{kg} \mathrm{N}\end{array}$

Estos valores son más altos que los del encinar de Rouquet, y muy parecidos a la media de los bosques de coníferas de la zona templada (Cole \& Rapp 1981). El encinar montano sería más eficaz en el uso del N que la media de los bosques caducifolios templados, lo cual representaría una ventaja en medios pobres o fluctuantes. No obstante, la eficiencia del hayedo de Santa Fe en el uso del $\mathrm{N}$ del desfronde, era mayor que la del encinar montano (Figura 1).

Las diferentes pautas de circulación de los distintos elementos en el encinar montano se pueden poner de manifiesto calculando para cada uno de ellos un índice de circulación dividiendo la mineralomasa aérea total por el retorno. Se obtiene así el tiempo de renovación o duplicación de cada nutriente. Los resultados son (años): Ca 23.8, P 17.4, Mg 14.3, 
$\mathrm{N} 12.3$, K 10.1, y Na 4.9. Estas cifras variarían un poco si tuviésemos en cuenta los flujos no medidos, pero son suficientemente indicativas. El comportamiento de $\mathrm{Ca}$ y $\mathrm{Na}$ es muy contrastado: el tiempo de renovación es menor cuando el nutriente se acumula en las fracciones de ciclo rápido ( $\mathrm{Na}$, en las hojas) que cuando lo hace en las de ciclo lento (Ca, en madera y corteza). No obstante, el tiempo de renovación del Na aumenta considerablemente si suponemos que el $\mathrm{Na}$ que se lava de las copas proviene de deposición seca y no de lixiviación. Resulta notable la similitud de los tiempos de renovación del $\mathrm{N}$ y del $\mathrm{K}$, que circulan de modos muy distintos: el $\mathrm{N}$ vuelve al suelo principalmente por el desfronde, mientras que el $\mathrm{K}$ lo hace a partes iguales por el desfronde y por la lixiviación. Sería de esperar que el $\mathbf{N}$ tuviese un menor tiempo de renovación, puesto que es el elemento que tiene un almacenamiento foliar más importante (19\% de la mineralomasa). Es probable que la retranslocación contribuya a alargar el tiempo de renovación del $\mathrm{N}$ en los árboles del encinar.

\section{BIBLIOGRAFIA}

ALLEN, S.E., CARLISLE, A., WHITE, E.J. \& EVANS, C.C. 1968. The plant nutrient content of rainwater. J. Ecol. 56: 497-504.

ANONIMO. 1976. Plan Especial del Parque del Montseny. I. Memoria informativa. Diputación de Barcelona, $90 \mathrm{pp}$.

ASTRUP, M. \& BULOW-OLSEN, A. 1979. Nutrient cycling in two Danish beech forests growing on different soil types. Holarctic Ecology 2: 125-129.

BOLOS, 0. de 1983. La vegetació del Montseny. Servei de Parcs Naturals, Diputació de Barcelona. Barcelona.

BRAY, J.R. \& GORHAM, E. 1964. Litter production in forests of the world. Adv. Ecol. Res. 2: $101-157$.

COLE, D.W. 1981. Nitrogen uptake and translocation by forest ecosystems, in: F.E. Clark \& T. Rosswall (eds.) Terrestrial Nitrogen Cycles. Ecol. Bull. (Stockholm) 33: 219-232.

COLE, D.W. \& RAPP, M. 1981. Elemental cycling in forest ecosystems, in: D.E. Reichle (ed.) Dynamic Properties of Forest Ecosystems. Cambridge University Press. Cambridge. 341-409 pp. 
DOCHINGER, L.S. \& SELIGA, T.A. (eds.) 1976. Proceedings of the First International Sympo sium on Acid Precipitacion and the Forest Ecosystem U.S.D.A. Forest Service General Technical Report NE-23.

DRABLOS, D. \& TOLLAN, A. (eds.) 1980. Ecological Impact of Acid Precipitation. Proc. Int. Conf. on the ecological impact of acid precipitation, SNSF project, Norwegian Institute for Water Research, Oslo.

EATON, J.S., LIKENS, G.E. \& BORMANN, F.H. 1973. Throughfall and stemflow chemistry in a northern hardwood forest. J. Ecol. 61: 495-508.

FARQUHAR, G.D. WETSELAAR, R. \& FIRTH, P.M. 1979. Ammonia volatilization from senescing leaves of maize. Science. 203: 1257-1258.

FERRES, Ll., RODA, F., VERDU, A.M.C. \& TERRADAS, J.A. 1980. Estructura y funcionalismo de un encinar montano en el Montseny. II. Biomasa arbórea. Mediterránea 4: 23-36.

FERRES, Ll., RODA, F., VERDU, A.M.C. \& TERRADAS, J.A. 1982. Nutrient cycling in a montane evergreen-oak forest at la Castanya (Montseny, Catalunya, NE Spain). U.S. Dep. Agric., For Serv. Gen. Tech. Rep. (PSW-58): 596.

GOSZ, J.B. 1981. Nitrogen cycling in coniferous ecosystems, in. F.E. Clark \& T. Rosswall (eds.) Terrestrial Nitrogen Cycles. Ecol. Bull. (Stockholm) 33: 405-426.

GRANAT, L. 1972. On the relation between $\mathrm{pH}$ and the chemical composition in atmospheric precipitation. Institute of Meteorology of the University of Stockholm \& International Meteorological Institute. Stockholm. 26 pp.

GRAY, J.T. 1983. Nutrient use by evergreen and deciduous shrubs in southern California. 1. Community nutrient cycling and nutrient-use efficiency. $J$. Ecol. 71: 21-41.

GRAY, J.T. \& SCHLESINGER, W.H. 1981. Nutrient cycling in mediterranean type ecosystems, in P.C. Miller (ed.) Resource Use by Chaparrat and Matorral. Springer-Verlag, New York, 259-285 pp.

HARWELL, M.A., CROPPER, W.P. \& RAGSDALE, H.L. 1977. Nutrient recycling and stability: a reevaluation. Ecology 58: 660-666.

HOFFMAN, W.A., Jr., LINDBERG, S.E. \& TURNER, R.R. 1980. Precipitation acidity: the role of the foresi canopy in acid exchange. J. Envir. Qual. 9: 95-100.

HUTCHINSON, T.C. \& HAVAS, M. (eds.) 1980. Effects of Acid Precipitation on Terrestrial Ecosystems. Plenum Press, New York.

KIRA, T. \& SHIDEI, T. 1967. Primary production and turnover of organic matter in different forest ecosystems on the western Pacific. Jap. J. Ecol. 17: 70-87.

KRAUSKOPF, K.B. 1979. Introduction to Geochemistry. McGraw-Hill, New York.

LEMÉE, G. 1978. La hétraie naturelle de Fontainebleau, in M. Lamotte \& F. Bourlière (eds.) Problèmes d'écologie: structure et fonctionnement des écosystèmes terrestres. Masson. Paris. 75-128 pp. 
LEONARDI, S. \& RAPP, M. 1980. Biomasse et composition minérale de Quercus ilex L. de Monte Minardo (Etna). Archivio Bot. Biogeogr. Ital. 56: 71-84.

LIKENS, G.E. \& BORMANN, F.H. 1974. Acid rain: A serious regional environmental problem. Science 184: 1176-1179.

LIKENS, G.E., BORMANN, F.H., JOHNSON, N.M., FISCHER, D.W. \& PIERCE, R.S. 1970. Effects of forest cutting and herbicide treatement on nutrient budgets in the Hubbard Brook watershed-ecosystem. Ecol. Monogr. 40: 23-47.

LikENS, G.E., WRIGHT, R.F., GAllowAY, J.N. \& BUTLER, T.J. 1979. Acid Rain. Sci. Amer. 241: 43-51.

LOSSAINT, P. \& RAPP, M. 1971. Répartition de la matière organique, productivité et cycles des éléments minéraux dans des écosystèmes du climat méditerranéen, in P. Duvigneaud (ed.) Productivity of Forest Ecosystems. Unesco. Paris. 597-617 pp.

LOSSAINT, P \& RAPP, M. 1978. La forêt méditerranéenne de chênes verts (Quercus ilex L.), in M. Lamotte \& F. Bourlière (eds.) Problèmes d'écologie; structure et fonctionnement des écosystèmes terrestres. Masson. Paris. 129-185 pp.

MATZNER, E., KHANNA, P.K., MEIWES, K.J., LINDHEIM, M., PRENZEL, J.\& ULRICH, B. 1982. Elementflüsse in Waldökosystemen im Solling. Datendokumentation. Göttinger Bodenkundliche Berichte 71: 1-267.

MECKLENBURG, R.A., TUKEY, H.B., Jr. \& MORGAN, J.V. 1966. A mechanism for the leaching of calcium from foliage. Plant Physiol. 41: 610-613.

NIHLGÅRD, B. 1970. Precipitation its chemical composition and effect on soil water in a beech and spruce forest in South Sweden. Oikos 21: 208-217.

NIHLGÅRD, B. \& LINOGREN, L. 1977. Plant biomass, primary production and bioelements of three mature beech forests in South Sweden. Oikos 28: 95-104.

OLSON, R.K., REINERS, W.A., CRONAN, C.S. \& LANGE, G.E. 1981. The chemistry and flux of throughfall and stemflow in subalpine balsam fir forests. Holarctic Ecology 4: 291-300.

O'NEILL, R.V., AUSMUS, B.S., JACKSON, D.R., VAN HOOK, R.I., VAN VORIS, P., WASHBURNE, C. \& WATSON, A.P. 1977. Monitoring terrestrial ecosystems by analysis of nutrient export. Water Air Soil Pollut. 8: 271-277.

OVERREIN, L.N. SEIP, H.M. \& TOLLAN, A. 1981. Acid precipitation - effects of forests and fish. Final report of the SNSF - project. 1972-1980. FR 19/80. Oslo.

PARKER, G.G. 1983. Troughfall and stemflow in the forest nutrient cycle. Avd. Ecol. Res. 13: 57-133.

RABElla, R., SAVE, R. \& TERRADAS, J.A. 1983. Perfiles verticales de conductancia estomática en Quercus ilex L. y Fagus sylvatical L. Seminario sobre Reservas de la Biosfera. Univ. Hispanoamericana de Sta. María de la Rábida (Huelva), 30 mayo-2 junio.

RAPP, M. 1969. Apport d'éléments minéraux au sol par les eaux de pluviolessivage sous des peuplements de Quercus ilex L., Quercus lanuginosa Lamk. et Pinus halepensis Mill. Oecol. Plant. 4: 71-92. 
RAPP, M. 1970. Cycle de la matière organique et des éléments minéraux dans quelques écosystèmes méditerranéens, in Ecologie du Sol. Recherche coopérative sur programme n ${ }^{\circ} 40$. C.N.R.S. Paris, vol. 2, 19-188 pp.

RODA, F. 1983. Biogeoquímica de les aigües de pluja i de drenatje d'alguns ecosistemes forestals del Montseny. Tesi Doctoral. Departament d'Ecologia. Universitat Autònoma de Barcelona. Bellaterra.

RODA, F., FERRES, LI., VERDU, A.M.C. \& TERRADAS, J.A. 1980. Estructura y funcionalismo de un encinar montano en el Montseny. III. Contenido de sodio, potasio, calcio, y magnesio en las aguas de la lluvia. Mediterránea 4: 37-56.

STEINHARDT, V. 1973. Input of chemical elements from the atmosphere. A tabular review of literature. Göttinger Bodenkundliche Berichte 29: 93-132.

SUSMEL, L., VIOLA, F. \& BASSATO, F. 1976. Ecologia della lecceta del Supramonte di Orgasolo (Sardegna Centro-orientale). Annali del Centro di Economia Montana delle Venezie, vol. $X$. 1-261 pp.

TERRADAS, J.A. \& ESCARRE, A. (dirs.) 1983. Ciclos de nutrientes en ecosistemas terrestres (bosques) estudiados en cuencas de pequeña extensión. Memòria final del Projecte de Recerca Cooperativa 0370, 1979-1983, 4 vols. Bellaterra (Barcelona).

TIEDEMANN, A.R., HELVEY, J.D. \& ANDERSON, T.D. 1980. Effects of chemical defoliation of an Abies grandis habitat on amounts and chemistry on throughfall and stemflow. J. Environ. Qual. 9: 320-328.

ULRICH, B. 1983. Ion cycle and forest ecosystem stability. Workshop on «Dynamics of Forest Ecosystems", European Science Foundation, Uppsala march 21-24.

ULRICH, B. \& PANKRATH, J. (eds.) 1983. Effects of accumulation of Air Polluntants in Forest Ecosystems. Reidel, Dordrecht.

Van VORIS, P., O'NEILL, R.V., EMANUEL, W.R. \& SHUGART, H.H., Jr. 1980. Functional complexity and ecosystem stability. Ecology 61: 1352-1360.

VERDU, A.M.C., FERRES, LI. RODA, F. \& TERRADAS, J. 1980. Estructura y funcionalismo de un encinar montano en el Montseny. IV. Producción de hojarasca. Mediterránea 4: 51-68.

VERRY, F.S. \& TIMMONS, D.R. 1977. Precipitation nutriens in the open and under two forests in Minnesota. Can. J. For. Res. 7: 112-119.

VITOUSEK, P. 1982. Nutrient cycling and nutrient use efficiency. Am. Nat. 119: 553-572.

WEBSTER, J.R. WAIDE, J.B. \& PATTEN, B.C. 1975. Nutrient recycling and the stability of ecosystems, in F.G. Howell, J.B. Gentry \& M.H. Smith (eds.) Mineral Cycling in Southeastern Ecosystems. Technical Information Center. Springfield, Virginia. 1-27 pp.

WHITEHEAD, H.C. \& FETH, G. 1964. Chemical composition of rain, dry fallout, and bulk precipitation at Menlo Park, California, 1957-1959. J. geophys. Res. 19: 3319-3333.

WOODCOCK, A.H. 1953. Salt nuclei in marine air as a function of altitude and wind force. $J . M e$ teorol. 10: $362-371$. 